\title{
RELATION OF POSTMORTEM CHANGES DEVELOPMENT AND EXACT POSTMORTEM INTERVAL
}

\author{
Grygorian E*., Olkhovsky V., Gubin M. \\ Kharkiv National Medical University, Ukraine \\ https://doi.org/10.35339/ic.7.2.72-75
}

\begin{abstract}
Purpose: Precise postmortem interval evaluation is crucial in cases when violent types of death are suspected by a forensic medical examiner. There are different factors that could affect results of postmortem interval (PMI) evaluation by a forensic medical expert. The aim of this study was to investigate the relationship between the known postmortem time interval and the degree of particular postmortem changes development. Materials and Methods: A cross-sectional analysis of 116 forensic medical examinations of deceased persons (of them, 58 females and 58 males), in cases of non-violent death, was performed. The data about the time of death was obtained from police preliminary records provided to the examination - only the cases with known time of death were included in the study. Postmortem changes were evaluated by Total Body Score (TBS) [1] at equal time interval after death (48 hours \pm 3 hours). Interconnection between postmortem changes degree and PMI was estimated using Spearman's rank correlation. Difference between sexes was evaluated using Mann-Whitney U test. Results: "Thickness of clothes" criterion reached the highest positive correlation coefficient, "ambient temperature" criterion had also a significant positive correlation. The rest of the studied criteria had very weak correlation with the development of postmortem changes. Conclusions: Several criteria had significant $(p<0.05)$, yet week, impact on the postmortem changes development. The other criteria were statistically insignificant.

Keywords: forensic medicine, forensic medical examination, postmortem interval, thanatology.
\end{abstract}

\section{Introduction}

Evaluation of the postmortem interval is one of the basic tasks of forensic medical expert. It plays a significant role in the work of police, due to a limited number of suspects who had an alibi when the crime occurred [2]. This evaluation is based on specific sequential changes that occur after death. The problem is that these postmortem changes vary due to many factors [3], including, but not limited to: ambient temperature, body weight, cloths, airflow [3, 4], and body length [5]. It could influence the estimation of PMI by a forensic medical practitioner, while performing examination [6], thus there is a need in defining

Corresponding Author:

Edgar Grygorian, MD, PhD student,

Department of Forensic Medicine, Medical Law,

Kharkiv National Medical University,

E-mail:8520148@gmail.com

${ }^{*}$ Part of PhD thesis by Edgar Grygorian the affective impact of these factors in order to further define new ways of making the PMI estimation more accurate as well as to elaborate methods which are already used by forensic medical experts.

\section{Purposes, subjects and methods:}

2.1. Purpose. The aim of this study was to define the level of interconnection between development of postmortem body changes and the time passed after the death. We hypothesized that: 1) there is strong interconnection between them which varies depending on the thickness of clothes, weight of the corpse, body length, and environmental factors - ambient temperature, airflow; 2) development of postmortem body changes does not depend on sex and/or age of deceased person.

\subsection{Subjects \& Methods \\ Design}

The study was based on 116 forensic medical examinations of deceased persons who died in a 
result of pathologies (non-violent conditions) performed within the time period of 2017-2018 at Regional Bureau of Forensic Medical Examinations. Exclusion criteria were violent cases of death (accidents, suicides, homicides), cases where the forensic medical diagnosis was not specified, e.g. due to putrid decomposition, and cases of pathological conditions which were accompanied by massive hemorrhages. The study was approved by the ethics committee of Kharkiv National Medical University in October 2018. The practical part of the study was finished by July, 2019. During the study, the following parameters were gathered and analyzed: sex, age, length of body, fat development level, peculiarities of scene, type of clothes on the corpse. Evaluation of postmortem changes was made using TBS in $48 \pm 3$, i.e. $46-51$, hours after the death. In all cases studied, the time of death was obtained from police preliminary reports provided to examination.

\section{Statistical Analysis}

Mean (M) and standard deviations (SD) were determined. In order to check distribution normality, Shapiro-Wilk test was performed. As far as the tests showed non-normality of distributions, Spearman's rank correlation was used to calculate connections between grade of postmortem changes and studied criteria, as well as in calculation of differences in female' and male postmortem changes development. For each test the significance level had been set as $\mathrm{p}<0.05$. All calculations were conducted using Microsoft Office Pro Plus 2019, StatSoft STATISTICA Version 8 (Tulsa, OK).

\section{Results}

The results were approximated to three decimal places.

The greatest correlation was observed in "thickness of clothes" criterion (Table 1, Figure 1). However, all the criteria studied had very weak or weak interconnections with the level of postmortem changes (i.e. less than 0.5 ), according to interpretation of Spearman rank correlation [7]. In particular, the mean of above mentioned "thickness of clothes" criterion was 0.320 - weak positive interconnection, "ambient temperature" criterion 0,249 (weak positive interconnection), "weight of corpse" criterion -0,055 (very weak negative interconnection), "body length" criterion $-0,088$ (very weak negative interconnection).

Analyzing and comparing outdoor and indoor scenes, it was found that, in cases with outdoor scenes, there was a weak positive correlation with postmortem changes development, while in cases with indoor scenes, there was a weak negative correlation. There was no information on the airflow level at the scene, so it was not possible to determine its impact on the postmortem changes`development.

Gender differences in females and males, in correlation with postmortem changes, were not significant $->0.1(p<0.05)$.

\section{Discussion}

Among the various methods of the postmortem interval evaluation, there is no specific one which could be precise enough to satisfy the needs of forensic medical examiners - to guarantee the adequate PMI evaluation in most of forensic medical examinations $[1,3,8]$. Scientists' examinations of pig carcasses as well as analysis of the investigations of human remains led to some controversial results. But even though some studies prove satisfactory results of currently used methods for some cases [3,9], there was one thing that was claimed in most studies - the methods should be modified in order to make the PMI evaluation more accurate $[1,3,10,11]$. Factors that should be evaluated are also varying in different studies, but there was no method that had gathered all factors affecting PMI and/or influence of these factors was not clearly measured [1, 3, 5-12].

Attempting to reach the maximum preciseness, the newly developed methods become more diverged from the currently used-e.g. bacteriologic tests [13], biochemical markers [14-20], immunohistochemistry [21,22]. These methods, on the one hand, show positive results in PMI evaluation. On the other hand, most of such methods are time consuming and, like those using

Table 1

The correlation between various factors and the postmortem changes development $(P M I=48$ hours \pm 3 hours $)$

\begin{tabular}{|c|c|c|c|c|}
\hline Age & Weight of the corpse & Body length & $\begin{array}{c}\text { Thickness } \\
\text { of clothes }\end{array}$ & $\begin{array}{c}\text { Ambient } \\
\text { temperature }\end{array}$ \\
\hline$<30$ & -0.081 & 0.017 & 0.231 & 0.215 \\
\hline $30-39$ & -0.002 & -0.251 & 0.363 & 0.150 \\
\hline $40-49$ & 0.077 & 0.062 & 0.229 & 0.314 \\
\hline $50-59$ & -0.154 & -0.147 & 0.420 & 0.312 \\
\hline$>59$ & -0.114 & -0.122 & 0.359 & 0.256 \\
\hline
\end{tabular}




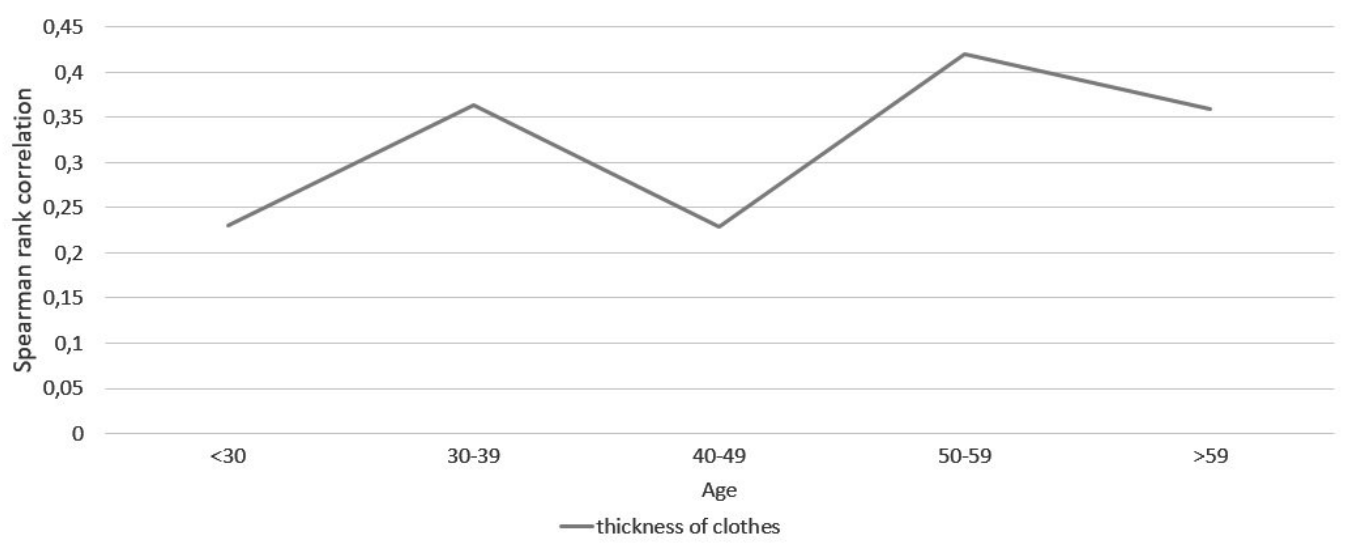

Figure 1. Spearman rank correlation of "thickness of clothes" criterion

immunohistochemistry, are expensive. Given the availability and quick assessment of PMI by currently used methods, such as TBS, it looks much more effective to supplement these methods first [23].

The limitation of the current study was the lack of complete information on the scene, which led to impossibility to measure all the additional factors affecting the postmortem changes of the body. Extensive data on scene peculiarities, such as airflow level, humidity, and also the more detailed information on the bedding of the corpse, sun exposure, could possibly let us to define their affection level on the PMI evaluation, with further comprehensive modifications to currently used methods.

\section{Conclusion}

During the study, it was found that there could be the impact of several factors on the development of postmortem changes. However, assuming the lack of information on the environment (airflow, humidity, sun exposure) in most studied cases, there is a need to evaluate the impact of these factors on the development of postmortem changes as well.

\section{Declaration}

The authors report no conflicts of interest.

\section{Data Availability}

The data that support the findings of this study are available from the corresponding author upon reasonable request.

\section{References}

1. Kim, Young et al. (2018). Feasibility of Total Body Score (TBS) and Accumulated Degree Days (ADD) in the Estimation of Postmortem Interval for Forensic Murder Casework. Biomedical Science Letters. 24. 35-42.

2. Max M.Houck, Jay A.Siegel (2010) Fundamentals of Forensic Science (Second Edition). 157-227

3. H. T. Gelderman, et al. (2018). The development of a post-mortem interval estimation for human remains found on land in the Netherlands. International journal of legal medicine. 132(3). 863-873.

4. C. Henssge, B. Madea (2004). Estimation of the time since death in the early post-mortem period. Forensic Science International. 144. 167-175.

5. Salimi M, et al. (2018) Evaluation of Insect Succession Patterns and Carcass Weight Loss for the Estimation of Postmortem Interval. J Med Entomol. Oct 25;55(6):1410-1422

6. Tsokos M. (2005) Postmortem Changes and Artifacts Occurring During the Early Postmortem Interval. In: Tsokos M. (eds) Forensic Pathology Reviews. Forensic Pathology Reviews, vol 3. Humana Press. 183-238.

7. Mukaka, M. M. (2012). Statistics Corner: A guide to appropriate use of Correlation coefficient in medical research. Malawi Medical Journal, 24(3), 69-71

8. Nawrocka, Marta \& Fratczak-Lagiewska, Katarzyna \& Matuszewski, Szymon. (2016). InterRater Reliability of Total Body Score-A Scale for Quantification of Corpse Decomposition. Journal of forensic sciences. 61 .

9. Dabbs GR, Connor M, Bytheway JA. (2016). Interobserver Reliability of the Total Body Score System for Quantifying Human Decomposition. J Forensic Sci. Mar;61(2):445-451.

10. Megyesi MS, Nawrocki SP, Haskell NH (2005) Using accumulated degree-days to estimate the postmortem interval from decomposed human remains. J Forensic Sci 50(3):618-626 
11. Devin Williams, Amy Mundorff, Audrey Scott (2016). Scoring Human Decomposition From Photographs: A Validation Study. EUReCA: Exhibition of Undergraduate Research and Creative Achievement. 4.

12. Lewis, Krystle \& Wescott, Daniel. (2018). Assessing the Utility of Total Body Score (TBS) and Accumulated Degree Days (ADD) for estimating Postmortem Interval (PMI) in Clothed Vulture-Scavenged Human Remains. 10.13140/RG.2.2.27264.12804.

13.Hurtado, J. C., et al. (2018). Postmortem Interval and Diagnostic Performance of the Autopsy Methods. Scientific reports, 8(1), 16112.

14. Meurs J, Krap T, Duijst W. (2019). Evaluation of postmortem biochemical markers: Completeness of data and assessment of implication in the field. Sci Justice. Mar;59(2):177-180.

15. Campell ZK, Kwon I, Finley SJ, Lee Y, Javan GT. (2016). Talin: A potential protein biomarker in postmortem investigations. J Forensic Leg Med. Nov;44:188-191.

16. Sara CZ, Menendez ST, Nunez P. (2014). Cell death proteins as markers of early postmortem interval. Cell Mol Life Sci. 71:2957-2962.

17. Kumar S, Ali W, Singh US, et al. (2015). The effect of elapsed time on the cardiac Troponin-T (cTnT) proteolysis in case of death due to burn: a study to evaluate the potential forensic use of cTnT to determine the postmortem interval. Sci Justice. 55:189-194.

18. Zheng XD, Zhang YH, Zhi XM, et al. (2006) Estimation of postmortem interval by determination of troponin I using Western blot technique in human pectoralis major. Chin J Forensic Med. 21:146-148.

19. Bian J, Shen YW, Zhao ZQ, et al. (2017) Relationship between the degradation of myoglobin and postmortem interval. Fa Yi Xue Za Zhi.23:90-91.

20. Sener MT et al. (2012). Estimating the postmortem interval by the difference between oxidant/ antioxidant parameters in liver tissue. Adv Clin Exp Med. Nov-Dec;21(6):727-33.

21. Lesnikova I., et al. (2018). Usability of Immunohistochemistry in Forensic Samples With Varying Decomposition. Am J Forensic Med Pathol. Sep;39(3):185-191.

22. LU Jiang-Ming, Yu Jia-Shu,Chen Min-Jing, et al. (2004). An immunohistochemical study on relationship between the changes of actin in cardiac and skeletal muscle cells and postmortem interval in rats. Chinese Journal of Forensic Medicine. 04.

23. Moffatt, Colin \& Simmons, Tal \& Lynch-Aird, Jeanne. (2016). An improved equation for TBS and ADD: establishing a reliable postmortem Interval framework for casework and experimental studies. Journal of Forensic Sciences. 61 Suppl 1. S201-207. 10.1111/1556-4029.12931.

Received: 08-Jan-2020

Accepted: 12-May-2020 